\title{
DETERMINACIÓN DE LA CURVA DE CRECIMIENTO EN OVINOS CRIOLLOS DE PELO, MEDIANTE LA UTILIZACIÓN DEL MODELO GOMPERTZ, EN EL TRÓPICO BAJO COLOMBIANO
}

\section{GROWTH CURVE DETERMINATION FOR CREOLE HAIR SHEEP USING THE GOMPERTZ MODEL IN THE LOW COLOMBIAN TROPIC}

\author{
Oscar Vergara Garay ${ }^{1}$, Hernán Medina Ríos ${ }^{2}$, César Robles Sierra ${ }^{3}$, Juan Simanca Sotelo ${ }^{4}$, Moris Bustamante Yanez $^{5}$
}

${ }^{1}$ Zoot, Dr. Sc. Grupo de Investigación en Producción Animal Tropical, Facultad de Medicina Veterinaria y Zootecnia. Universidad de Córdoba. Montería, Colombia, e-mail: overgara@correo.unicordoba.edu.co; ${ }^{2}$ MVZ, Grupo de Investigación en Producción Animal Tropical, Facultad de Medicina Veterinaria y Zootecnia. Universidad de Córdoba, Montería, Colombia, e-mail: hernanmedinarios@gmail.com; ${ }^{3}$ MVZ. Grupo de Investigación en Producción Animal Tropical, Facultad de Medicina Veterinaria y Zootecnia. Universidad de Córdoba, Montería, Colombia, e-mail: cars_mvz@ hotmail.com; ${ }^{4}$ MVZ, M.Sc. Maestría en Ciencias Veterinarias del Trópico, Facultad de Medicina Veterinaria y Zootecnia. Universidad de Córdoba, Montería, Colombia, e-mail: j.c.simanca@hotmail.com; ${ }^{5}$ MVZ, M.Sc. Maestría en Ciencias Veterinarias del Trópico, Facultad de Medicina Veterinaria y Zootecnia. Universidad de Córdoba, Montería, Colombia, e-mail: morisbustamante@correo.unicordoba.edu.co

Rev. U.D.C.A Act. \& Div. Cient. 20(2): 385-391, Julio Diciembre, 2017

\section{RESUMEN}

En Colombia existe una diversidad de ovinos, denominados ovinos criollos, resultado del cruce de diferentes razas, desde la época de la colonización y que se adaptaron a diferentes regiones del país. En el caso particular de este estudio, se hace referencia a los ovinos criollos de pelo, que se acomodaron a las condiciones biogeográficas de la Costa Caribe colombiana. Estos animales, por su adaptabilidad, han tenido un importante papel en el desarrollo de la ovinocultura del país; sin embargo, es muy poca la información que se tiene, a nivel nacional, sobre las características crecimiento de la raza o especie. El objetivo del presente estudio fue determinar la curva de crecimiento de dos poblaciones de ovinos criollos del trópico bajo colombiano, a través del uso del modelo no lineal Gompertz. Se utilizaron 55 animales, con 13 pesajes cada uno, perteneciente a dos sistemas de producción del departamento de Córdoba, Colombia. Se realizó un ajuste del modelo, mediante el procedimiento NLIN de Statistical Analysis Software (SAS), para la estimación de los parámetros del modelo. Se estimó $\beta_{0}, \beta_{1}$ y $\beta_{2}$, cuyos valores fueron $25,97 \pm 9,3,2,1 \pm 0,5$ y $0,010 \pm 0,004$, respectivamente. Los estimados de madurez a los 4 y 6 meses fueron de 55,8 y $70,6 \%$, respectivamente, y la edad al 75 de madurez fue de 7,0 meses y al $95 \%$ de madurez, de 13,1 meses. El modelo de Gompertz permitió describir el crecimiento de ovinos criollos, en condiciones de pastoreo, en el trópico bajo.
Palabras clave: Criollos, madurez, pastoreo, trópico.

\section{SUMMARY}

The Colombian Creole sheep has an important role in the development of sheep farming in the country, as this breed is distributed in most of the territory, being animals with significant features that make it widely used in farms with different productive purposes. To assess growth characteristics in animals have been used mathematical models, which describe the relationship between the age of the animal, its rate of growth and maturity. These models are equations that allow the construction of continuous curves of one biological variable according to another. This study aimed to determine the growth curve of two populations of crossbred sheep through the use of nonlinear Gompertz model. 55 animals with 13 measures of weight each were used, pertaining to two production systems of the department of Córdoba, Colombia. Estimaste for $\beta_{0}, \beta_{1}$ y $\beta_{2}$ were $25.97 \pm 9.3,2.1 \pm$ 0.5 and $0.010 \pm 0.004$, respectively. Estimates of maturity at 4 and 6 months were 55.8 and $70.6 \%$, respectively; age $75 \%$ of maturity was of 7.0 months and at $95 \%$ of maturity was of 13.1 months. Gompertz model allowed describing the growth of creole sheep in conditions of grazing in the low tropics.

Key words: Creoles, grazing, maturity, tropics. 


\section{INTRODUCCIÓN}

En los sistemas de producción ganaderos, cuyo objetivo principal es la producción de alimentos para el consumo humano, interfieren muchos factores que interactúan entre sí, partiendo desde el productor primario hasta los canales de comercialización, para llegar el productor final (Lozano, 2014), donde las políticas gubernamentales y el desarrollo tecnológico local son fundamentales, para lograr el desarrollo productivo. A pesar que en Colombia la mayoría de los sistemas de producción ovina están en manos de pequeños productores, que tienen poco acceso a la tecnología, éstos juegan un papel preponderante en la economía familiar (Acero-Plazas, 2014). Estos sistemas de producción, se caracterizan por presentar prolificidad, de 1,0 a 1,2; peso al nacer, de 3,0 a 3,3kg y peso al destete, de 12,3 a 18,0kg (Martínez \& Malagón, 2005; Martínez \& Vásquez, 2005; Duque \& Chamorro, 2011).

Partiendo de lo anterior, es ideal plantear programas de mejora genética. que cumplan con las necesidades del productor. mejorando los índices de parámetros productivos y reproductivos. para obtener un producto de mejor calidad y competir con mercados internacionales. Para mejorar la producción ovina a futuro en el país, se debe conocer y tener en cuenta tres aspectos fundamentales: eficiencia reproductiva, velocidad del crecimiento y la calidad de la canal, por lo tanto, el crecimiento de los animales reviste importancia al ser medido, a través de modelos matemáticos, que predicen el desempeño de la evolución del peso vivo (Patiño \& Van Cleef, 2010).

Estas funciones o modelos matemáticos permiten realizar evaluaciones sobre el nivel de producción en las empresas ganaderas, pudiendo clasificar, de forma sencilla, la productividad de una raza específica, para una zona determinada y, además, evalúan parámetros biológicamente importantes, dependiendo del modelo matemático utilizado (Agudelo et al. 2008). De igual manera, mediante las curvas de crecimiento, se pueden ver reflejadas la relación entre la edad del animal y el impulso propio del individuo, para crecer y desarrollarse en el medio donde se encuentran (Fitzhugh, 1976), por lo que su conocimiento es importante en el monitoreo de un hato, ya que ellas expresan gran cantidad de información inherentes al animal, durante toda su vida.

En este sentido, en Colombia es escasa la información de las características zootécnicas de importancia económica en los ovinos producidos bajo condiciones tropicales, sobre todo los ovinos de pelo, por lo que se propuso, como objetivo de este estudio, determinar la curva de crecimiento en ovinos criollos de pelo, en condiciones de pastoreo, en dos poblaciones ovinas, del departamento de Córdoba.

\section{MATERIALES Y MÉTODOS}

Localización. Para la realización de este trabajo, se utilizaron las bases de datos de dos sistemas productivos del Departamento de Córdoba, ubicados en los municipios de Montería (Finca 1) y Sahagún (Finca 2). Montería, se encuentra ubicado a $08^{\circ} 45^{\prime}$ latitud Norte y $75^{\circ} 53^{\prime}$ longitud Oeste, a una altura de 18 m.s.n.m., temperatura media anual de $28^{\circ} \mathrm{C}$, precipitación media anual de $1225 \mathrm{~mm}$ y humedad relativa de $80 \%$. El municipio de Sahagún está ubicado al nororiente del departamento de Córdoba, a $08^{\circ} 56^{`}$ latitud Norte y $75^{\circ}$ 26 longitud Oeste, a una altura de 82 m.s.n.m.; este municipio presenta una temperatura promedio anual de $27^{\circ} \mathrm{C}$, humedad relativa del $80 \%$ y una precipitación promedio de $1.249 \mathrm{~mm}$ anuales. Las precipitaciones en el departamento están marcadas desigualmente en dos periodos: uno lluvioso, en los que caen $85 \%$ del total de la precipitación anual, comprendido desde mayo a octubre y un periodo seco, desde noviembre hasta abril (Santana, 1999).

Datos y animales. Para la realización de este estudio, se utilizaron 55 animales, 15 en el sistema de producción de Montería (9 machos y 6 hembras) y 40, en el sistema de producción de Sahagún (18 machos y 22 hembras); los animales nacieron entre noviembre del 2013 y abril del 2014. Estos animales fueron pesados cada 15 días, desde el nacimiento hasta los seis meses de edad; el destete, se realizó a los 90 días. Los animales fueron pesados al nacer y después se realizaban pesajes cada 15 días. Todos los animales provenían de partos sencillos y no se tenía información precisa sobre el número de partos de la madre. Cada sistema de producción dispuso de una báscula para efectuar los pesajes de los animales, los cuales, fueron anotados en registros, diseñados para tabulación de estos datos. Igualmente, en los registros se anotaron las fechas y las edades de los animales al momento de los pesajes y el número de animales. Los animales fueron manejados bajo condiciones de pastoreo, en praderas de Bothriochloa pertusa, con disponibilidad de agua y sal mineral a voluntad; durante la época seca, los animales del sistema de producción de Montería, se suplementaron con, aproximadamente, 500gr de ensilaje de maíz (Zea mays) y los del sistema de producción de Sahagún, con ensilaje de Pennisetum purpureum, a voluntad.

Análisis de datos. La información de campo fue digitada en una base de datos de Excel. El modelo no lineal que se utilizó para describir el crecimiento fue Gompertz, cuya ecuación fue la siguiente:

$$
y_{i}=\beta_{0} e^{-\beta_{1} e^{-\beta_{2} t_{i}}}, \text { Gompertz (1825) }
$$

Donde: $y_{i}$ representa el i-ésimo peso del animal en el i-ésimo tiempo ti; $\beta$, es el peso asintótico cuando $t$ tiende a infinito; 
$\beta_{1}$ es un parámetro de ajuste cuando $y \neq 0$ o $t \neq 0 ; \beta_{2}$ es un índice de madurez expresado como una proporción de porcentaje del máximo crecimiento con respecto al peso adulto del animal.

Fueron validados los supuestos de normalidad e independencia de los errores. Para la estimación de los parámetros del modelo, se utilizó el procedimiento NLIN de SAS. Además, se calculó el porcentaje de madurez a los cuatro (M4) y seis meses (M6) y edad al 75 (EM75) y 95\% (EM95) de madurez, para las poblaciones ovinas estudiadas, mediante las siguientes expresiones:

$$
\begin{aligned}
& M 4=e(-\beta 1 * e(-\beta 2 * 120)) \\
& M 6=e(-\beta 1 * e(-\beta 2 * 180)) \\
& E M 75=\frac{\log \left(-\log (0.75) / \beta_{1}\right.}{-\beta_{2}} \\
& E M 95=\frac{\log \left(-\log (0.95) / \beta_{1}\right.}{-\beta_{2}}
\end{aligned}
$$

Para determinar el efecto sexo y finca sobre los parámetros del modelo y las edades y porcentajes de madurez, se aplicó un análisis de varianza, el cual, se realizó mediante el procedimiento GLM, de SAS (2002).

\section{RESULTADOS Y DISCUSIÓN}

El estimado para $\beta_{0}, \beta_{1}$ y $\beta_{2}$ fueron $25,57 \pm 9,3,2,1 \pm 0,5$ y $0,010 \pm 0,004$, respectivamente. El modelo presentó un $R^{2}$ de 0,994 . En la figura 1, se observa la curva de crecimiento general de los ovinos criollos de pelo, de acuerdo con los parámetros del modelo de Gompertz.

Con relación a $\beta$, Lupi et al. (2015) reportaron valores superiores a los encontrados en este estudio para machos (36,41 $\pm 12,3)$ y para hembras $(36,12 \pm 11,4)$, datos similares a los encontrados por Moreira et al. (2016), Santos (2012) y Teixeira et al. (2016), con estimados de 45,43, 42,41 y 37,56, respectivamente. Por su parte, Méndez et al. (2014) evaluaron la curva de crecimiento en borregos de la raza Chiapas, en México, obteniendo valores de 25,4 y 24,8, para machos y hembras, respectivamente; Bahreini et al. (2014), en la raza Baluchi y Kopuzlu et al. (2014), en la raza Hemsin, reportaron valores, para machos, de 43,07 y 74,99 y para hembras,

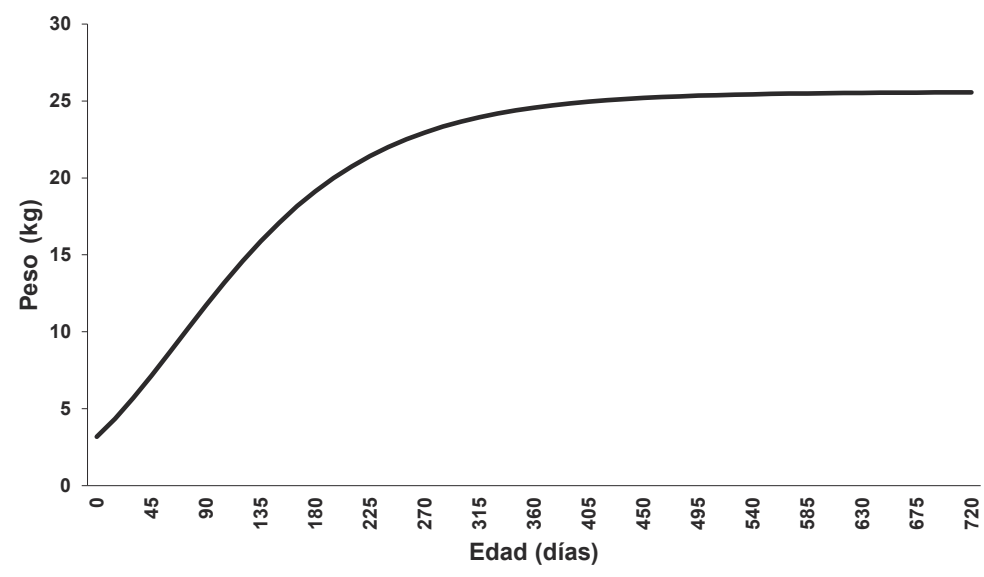

Figura 1. Curva de crecimiento en ovinos criollos, de acuerdo al modelo de Gompertz.

de 39,68 y 58,77, respectivamente. El valor de $\beta_{0}$ está relacionado con el peso del animal adulto, que va a depender de diversos factores, como son la especie, la raza, el método de selección, el sistema de manejo y las condiciones ambientales (Malhado et al. 2009).

Respecto al valor estimado para $\beta_{1}$, Hossein-Zadeh \& Golshani (2016), en la raza Iraní Guilan y Moreira et al. (2016), en la raza Lle de Francia, encontraron valores similares al de este estudio (2,10 y 2,11), mientras que Lupi et al. (2015) y Méndez et al. (2014) reportaron valores, para machos, de 2,27 y 2,09 y para hembras de 2,25 y 1,90, en las razas
Segureña y Chiapas, respectivamente, valores cercanos al encontrado por este estudio; sin embargo, Da Silva et al. (2012), en ovinos Santa Inés, Santos (2012), en animales cruzados Santa Inés por Dorper y Sieklicki et al. (2016), en la raza Texel, registraron valores inferiores $(-1.62,0,71$ y 1,95 , respectivamente). Varios autores coinciden en decir que este parámetro no posee interpretación biológica; no obstante, Abreu et al. (2004) afirma que es un factor de integración que ajusta los valores de peso inicial y, generalmente, está asociado con el peso al nacimiento (grado de madurez del animal al nacimiento). 
Por su parte, el estimado para $\beta_{2}$ indica la tasa de madurez del animal y entre más alto sea este parámetro, más rápido llega el animal al peso adulto. Animales con altos valores de $\beta_{2}$ tienen una madurez precoz, en relación a los que presentan bajos valores y un peso inicial similar, por lo tanto, animales que tienen un crecimiento más rápido pueden ser sacrificados a una menor edad (Malhado et al. 2009). Valores superiores fueron reportados por Lupi et al. (2015), cuyos valores fueron de 0,022 y 0,020, para machos y hembras, respectivamente; sin embargo, valores inferiores fueron hallados en los estudios adelantados por Da Silva et al. (2012), donde el valor encontrado fue de 0,004; Méndez et al. (2014), con un estimado de 0,0056, mientras que Hossein-Zadeh \& Golshani (2016), Moreira et al. (2016), Santos (2012) y Teixeira et al. (2016) reportaron valores similares al determinado en este estudio, donde los valores estimado por los autores, anteriormente mencionados, fueron de 0,015, 0,016, 0,009 y 0,015 , respectivamente. Al momento de realizar la comparación de los valores reportados por otros autores y los estimados por nuestro estudio, se puede concluir, que los corderos criollos de pelo estudiados demostraron que tienen una precocidad muy baja, por lo tanto, la velocidad de crecimiento se verá afectada, debido a la lentitud de los animales para llegar al peso asintótico.

Sarmento et al. (2006), comparando funciones en la curva de crecimiento en ovinos de la raza Santa Inés concluyeron que el modelo de Gompertz ofrece resultados más fiables, similar a lo reportado por Lambe et al. (2006), quienes consideran que este modelo presenta los mejores ajustes para describir el peso, desde el nacimiento hasta el sacrificio, en las razas Texel y Blackface.

De lo anterior, se puede deducir, que animales con madurez tardía o una tasa de madurez baja, ocasionan un retraso en el sistema de producción, debido a que esos corderos tomarán más tiempo para llegar al peso al sacrifico, por lo que es de suma importancia para el ovinocultor conocer a qué edad sus animales llegan a la madurez y tratar de reducirla, puesto que una mayor tasa de crecimiento, se asocia a un aumento de la rentabilidad económica de la producción.

Respecto al porcentaje de madurez a los cuatro meses (M4) y seis meses (M6) (Tabla 1), se puede considerar que, de estas variables, la más importantes es M6, la cual, es la edad que los productores tienen como referencia en los rebaños de la región para llevar los corderos a sacrificio o destinar las hembras aptas para la reproducción, como hembras de vientres. Vergara et al. (2016), en ovinos criollos, reportaron edades al 75 y $95 \%$ de madurez, superiores a las de este estudio (9,0 y 19,5 meses, respectivamente). Por su parte, Simanca et al. (2016) reportaron en ovinos Santa Inés, por criollo, un promedio de edad al $75 \%$ de madurez, de 10,2 meses y al 95\% de madurez, una edad promedio de 19,3 meses. De acuerdo con los porcentajes de madurez y las edades al 75 y $95 \%$ de madurez estimados, es pertinente hacer algunos correctivos en los sistemas de alimentación, implementados en los sistemas de producción y en el manejo reproductivo de los animales.

En la tabla 2, se observan los promedios, de acuerdo al sexo y a la finca de las poblaciones estudiadas, según el modelo Gompertz; se evidenció que el sexo no tuvo efectos significativos sobre las variables estudiadas. Malhado et al. (2009) encontraron que el sexo del cordero no presentó efectos significativos sobre $\beta_{0}$, a diferencia de Sarmento et al. (2006) y Keskin et al. (2009), quienes reportaron que los machos presentan mayores valores de $\beta_{0}$. En la figura 2 , se detalla la curva de crecimiento de los ovinos criollos, según el modelo Gompertz, de acuerdo al sexo y a la finca, en la cual, se puede observar el comportamiento similar de machos y de hembras y el mejor desempeño de la Finca 1, respecto a la Finca 2. La variación del crecimiento de los animales entre fincas puede estar determinado por la diferencia en el siste-

Tabla 1. Estimados del porcentaje de madurez a los 4 y 6 meses y edades al 75 y $95 \%$ de madurez, en las poblaciones ovinas en estudio.

\begin{tabular}{|l|c|c|}
\hline \multicolumn{1}{|c|}{ Variable } & Promedio & Desviación estándar \\
\hline M4 (\%) & 55,8 & 18,2 \\
\hline M6 (\%) & 70,6 & 18,5 \\
\hline EM75 (meses) & 7,0 & 3,5 \\
\hline EM95 (meses) & 13,1 & 6,0 \\
\hline
\end{tabular}

M4 = porcentaje de madurez a los 4 meses; $M 6=$ porcentaje de madurez a los 6 meses; EM75= Edad al alcanzar el 75\% de madurez; EM95= Edad al alcanzar el 95\% de madurez. 
ma de alimentación de cada finca y la selección fenotípica, con base en los registros productivos que se práctica en la Finca 1.

Por último, se puede afirmar que, al utilizar el modelo Gompertz en la descripción de la curva de crecimiento de ovinos, resulta eficiente, para establecer predicciones en el comportamiento productivo, reflejado en el futuro inicio de la actividad reproductiva de nuevas ovejas, candidatas a ser vientres de reemplazo. Además, el modelo de Gompertz permitió describir el crecimiento de ovinos criollos, de acuerdo con las características de crecimiento en el trópico, con ali- mentación basada en pastoreo; el tipo de curva, se ajustó al sentido estricto de la curva sigmoidea, característica de los modelos no lineales.

Agradecimientos. Los autores agradecen a Mauricio Buelvas y Rafael Vergara, propietarios de los sistemas de producción, por facilitar los animales e instalaciones para la realización de este estudio. Conflicto de intereses: El manuscrito fue preparado y revisado con la participación de todos los autores quienes declaramos que no existe conflicto de intereses que ponga en riesgo la validez de los resultados presentados.

Tabla 2. Valores promedios estimados para los parámetros del modelo Gompertz y edades y porcentajes de madurez, según el sexo y la finca.

\begin{tabular}{|l|c|c|c|c|c|c|c|c|}
\hline \multirow{2}{*}{ Efecto } & & $\beta_{0}$ & $\beta_{1}$ & $\beta_{2}$ & \%M4 & \%M6 & $\begin{array}{c}\text { EM75\% } \\
\text { (días) }\end{array}$ & $\begin{array}{c}\text { EM95\% } \\
\text { (días) }\end{array}$ \\
\hline \multirow{2}{*}{ Sexo } & Macho & 26,08 & 1,99 & 0,013 & 63,3 & 77,3 & 180 & 341 \\
\cline { 2 - 10 } & Hembra & 26,65 & 2,19 & 0,011 & 54,5 & 70,1 & 211 & 389 \\
\hline \multirow{2}{*}{ Finca } & 1 & 28,12 & 2,10 & $0,014^{\mathrm{a}}$ & $65,5^{\mathrm{a}}$ & $80,2^{\mathrm{a}}$ & $161^{\mathrm{a}}$ & $299^{\mathrm{a}}$ \\
\cline { 2 - 10 } & 2 & 24,61 & 2,08 & $0,009^{\mathrm{b}}$ & $52,3^{\mathrm{b}}$ & $67,1^{\mathrm{b}}$ & $230^{\mathrm{b}}$ & $431^{\mathrm{b}}$ \\
\hline
\end{tabular}

Letras diferentes en la misma columna, indica diferencia significativa $(\mathrm{p} \leq 0,05)$.

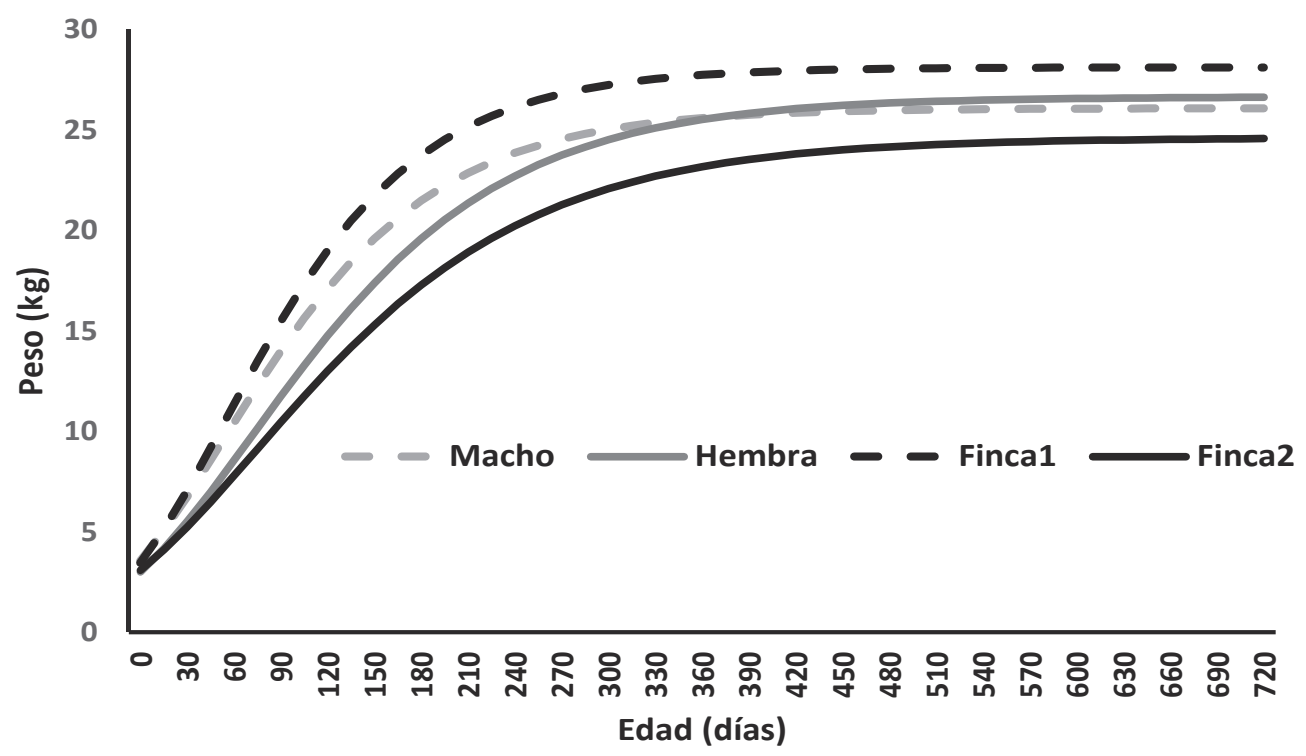

Figura 2. Curva de crecimiento de ovinos criollos, según el modelo Gompertz, de acuerdo al sexo y a la finca de las poblaciones estudiadas. 


\section{BIBLIOGRAFÍA}

1. ABREU, U.; COBUCI, A.; DA SILVA, M.; SERENO, J. 2004. Uso de modelos no lineales para el ajuste de la curva de crecimiento de bovinos Pantaneiros. Arch. Zootec. 53(204):367-370.

2. ACERO-PLAZAS, V.M. 2014. El bienestar animal en sistemas productivos de ovinos-caprinos en Colombia. Spei Domus. 10(20):57-62.

3. AGUDELO, D.; CERÓN, M.; RESTREPO, L. 2008. Modelación de las funciones de crecimiento aplicadas a la producción animal. Rev Col. Cienc. Pec. 21(1):3958.

4. BAHREINI, M.R.; ASLAMINEJAD, A.A.; SHARIFI, A.R.; SIMIANER, H. 2014. Comparison of mathematical models for describing the growth of Baluchi sheep. J. Agric Sci and Technol. 14:57-68.

5. DA SILVA, L.; FRAGA, A.; DA SILVA, F.; BEELEN, P.; SILVA, R.; TONHATI, H.; BARROS, C. 2012. Growth curve in Santa Inês sheep. Small Rumin. Res. 105:182-185.

6. DUQUE-CHAMORRO, L.M.; PULGARÍN-VERGARA, L. 2011. Evaluación reproductiva y productiva en un rebaño de ovejas (Ovis orientalis aries) en el municipio de Bello, Antioquia. Rev Col. Cienc. Pec. 24(3):547.7.

7. FITZHUGH, H. 1976. Analysis of growth curves and strategies for altering their shapes. J. Anim. Sci. 42(4):1036-1051.

8. GOMPERTZ, B. 1825. On the nature of the function expressive of the law of human mortality, and on a new Mode of determining the value of life Contingencies. Phil. Trans Royal Soc London. 115:513-585.

9. HOSSEIN-ZADEH, N.; GOLSHANI, M. 2016. Comparison of non-linear models to describe growth of Iranian Guilan sheep. Rev. Col. Cienc. Pec. 29:199-209.

10. KESKIN, I.; DAG, B.; SARIYEL, V.; GOKMEN, M. 2009. Estimation of growth curve parameters in Konya Merino sheep. J. Anim. Sci. 39(2):163-168.

11. KOPUZLU, S.; SEZGIN, E.; ESENBUGA, N.; BILGIN, O. 2014. Estimation of growth curve characteristics of Hemsin male and female sheep. J. Applied Anim. Res. 42(2):228-232.
12. LAMBE, N.R.; NAVAJAS, E.A.; SIMM, G.; BÜNGER, L. 2006. A genetic investigation of various growth models to describe growth of lambs of two contrasting breeds. J. Anim. Sci. 84:2642-2654.

13. LOZANO, H. 2014. Reproducción ovina en Colombia. Rev. Ciencia Ani. 8:67-83.

14. LUPI, T.; NOGALES, S.; LEÓN, J.; DELGADO, J. 2015. Modelación de curvas de crecimiento comercial en ovino Segureño. AICA. 6:132-143.

15. MALHADO, C.; CARNEIRO, P.; AFFONSO, P.; SOUZA, A.; SARMENTO, J. 2009. Growth curves in Dorper sheep crossed with the local Brazilian breeds, Morada Nova, Rabo Largo, and Santa Inês. Small Rum. Res. 84:16-21.

16. MARTÍNEZ, R.; MALAGÓN, S. 2005. Caracterización fenotípica y genética del ovino criollo colombiano. Arch. Zootec. 54:341-348.

17. MARTÍNEZ, R.; VÁSQUEZ, R. 2005 Evaluación de la conservación y comportamiento productivo del Banco de Germoplasma de la especie ovina en Colombia. AGRI. 36:33-45.

18. MÉNDEZ, A.; LÓPEZ, R.; PERALTA, M.; ULLOA, R.; PEDRAZA, P.; RUIZ, F.; BERRUECOS, J.; VÁSQUEZ, C. 2014. Estimación de heredabilidad de la curva de crecimiento en el borrego de raza Chiapas en México. Anim. Gen. Resour. 54:85-91.

19. MOREIRA, R.; PEDROSA, V.; FALCAO, P.; SIEKLICKI, M.; ROCHA, C.; DOS SANTOS, I.; FERREIRA, E.; MARTINS, A. 2016. Curvas de crescimento de fêmeas da raça Lle de France criadas em confinamento. Ciências Agrárias. 37(1):303-310.

20. PATIÑO, R.; VAN CLEEF, E. 2010. Aspectos chave do crescimento em ovinos. Revisión. Rev. Colombiana Cienc. Anim., 2(2): 399-421.

21. SANTANA, V. 1999. Diccionario Cultural de Córdoba. Domus Libri. Bogotá (Colombia). p. 285-291.

22. STATISTICAL ANALYSIS SYSTEM INSTITUTE -SAS-. 2002. SAS/STAT User's guide (Relase 9.1.3), Cary, NC, USA.

23. SANTOS, S. 2012. Crescimento de ovinos de diferentes grupos genéticos sob modelos não lineares convencionais e alternativos. Disponible desde Internet 
en: http://www.uesb.br/ppz/defesas/2012/mestrado/ lorena-mirelle.pdf (con acceso 04/08/2017).

24. SARMENTO, J.; REGAZZI, A.; DE SOUSA, W.; TORRES, R.; BREDA, F. 2006. Estudo da curva de crescimento de ovinos Santa Inês. R. Bras. Zootec. 35(2):435442.

25. SIEKLICKI, M.F.; PEDROSA, V.B.; ROCHA, C.G.; MOREIRA, R.P.; FALCAO, P.R.; SANTOS, I.C.; FERREIRA, E.M.; MARTINS, A. 2016. Growth curves of Texel male lambs. Acta Scientiae Vet. 44(1396):1-6.26. SIMANCA, J.; VERGARA, O.; BUSTAMANTE, M. 2016. Descripción del crecimiento de ovinos Santa Inés $x$ Criollo manejados en pastoreo extensivo en dos poblaciones de Córdoba, Colombia. Rev. Fac. Cs. Vets. UCV. 57(1):61-67.
27. TEIXEIRA, M.R.; CRUZ, J.F.; FARIA, H.H.; SOUZA, E.S.; CARNERO, P.L; MALHADO, C.H. 2016. Descrição do crescimento de ovinos Santa Inês utilizando modelos não-lineares selecionados por análise multivariada. Rev. Bras. Saúde Prod Anim. 7(1):26-36.

28. VERGARA-GARAY, O.; LLORENTE-MARTÍNEZ, E.; RAMOS-CARO, L.; BUSTAMANTE-YANEZ, M.; SIMANCA-SOTELO, J. 2016. Descripción del crecimiento en ovinos criollos utilizando el modelo Brody. Rev. Orinoquía. 20(2):34-39.

Recibido: Mayo 25 de 2017

Aprobado: Octubre 2 de 2017

Cómo citar:

Vergara Garay, O.; Medina Ríos, H.; Robles Sierra, C.; Simanca Sotelo, J.; Bustamante Yanez, M. 2017. Determinación de la curva de crecimiento en ovinos criollos de pelo, mediante la utilización del modelo Gompertz, en el trópico bajo colombiano. Rev. U.D.C.A Act. \& Div. Cient. 20(2): 385-391. 